\title{
The research on plasma nitriding of AISI410 martensitic stainless steel \\ Xingsheng Tong ${ }^{a}$, Chen Wang ${ }^{b}$, Wei Ye
}

School of Electro-mechanical and Architectural Engineering Jianghan University,

Wuhan 430056, China

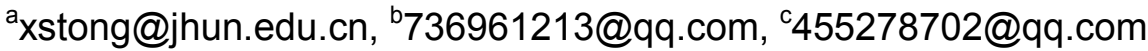

Keywords: martensitic stainless steel; plasma nitriding; hardness; microstructure

Abstract. In this study, the AISI410 martensitic stainless steel was treated by plasma nitriding at different temperature, different time and different gas proportion. Then the hardness and microstructure were studied by a variety of analytical techniques. The results show that the hardness of nitrided layer decreases and the thickness of nitrided layer enhances with the improvement of temperature; When nitrided time increases, both of the hardness and thickness improve. However, when to increase gas proportion, the hardness of surface reduces and the thickness of nitrided layer almost still remains the same.

\section{Introduction}

The martensitic stainless steel are often used in fabricating components requiring highmechanical properties and moderate corrosion resistance, such as cutting tools, valve parts, cutlery and fasteners. But its poor hardness and corrosion property limits the parts of life.

Plasma nitriding as a surface modification technology that can effectively improve the hardness of the surface of the part, wear resistance and corrosion resistance ${ }^{[1,2]}$. For example, nitriding at a temperature around $500^{\circ} \mathrm{C}$ can produce a thick nitrided case on an austenitic stainless steel surface, which significantly improves the surface hardness and wear properties ${ }^{[3-5]}$.

This paper mainly focuses on changes of the hardness and microstructure of plasma nitrided martensitic stainless at different temperature, different time and different gas proportion.

\section{Experimental}

The material used in the study was an AISI410 martensitic stainless steel with the size of $\phi 8 \mathrm{~mm} \times$ $15 \mathrm{~mm}$. The samples were plasma nitrided with the LDMC-20A double glow heat treatment furnace with $\mathrm{Ar}$ and $\mathrm{NH}_{3}$. Specific experimental parameters were shown in Table 1.

The hardness of the samples was measured with HVS-10-micro-hardness tester when the loading force was $9.8 \mathrm{~N}$ for $15 \mathrm{~s}$. Using 1-6 metallographic sandpaper to uniform the sample surface and obtain a smooth surface without scratches for metallographic experiments. Use $5 \mathrm{~g} \mathrm{FeCl}, 50 \mathrm{ml}$ $\mathrm{HCl}$, and $100 \mathrm{ml} \mathrm{H}_{2} \mathrm{O}$ as ferric chloride hydrochloric reagent to reveal the martensitic grains. 


\begin{tabular}{|c|c|c|c|}
\hline Number & Temperature $\left({ }^{\circ} \mathrm{C}\right)$ & Time $(\mathrm{h})$ & Gas Proportion \\
\hline 1 & 420 & 6 & $8: 1$ \\
\hline 2 & 460 & 6 & $8: 1$ \\
\hline 3 & 500 & 6 & $8: 1$ \\
\hline 4 & 420 & 9 & $8: 1$ \\
\hline 5 & 420 & 12 & $8: 1$ \\
\hline 6 & 420 & 6 & $12: 1$ \\
\hline 7 & 420 & 6 & $16: 1$ \\
\hline
\end{tabular}

Table 1. Experimental parameters of low temperature plasma nitriding

\section{Results and analysis}

Hardness Analysis. The Fig 1(a) shows the hardness of the nitrided layer at different temperature. The hardness decreases with the temperature improves and it becomes highest at $420^{\circ} \mathrm{C}$ that is different from tendency of austenitic stainless steel. The hardness is beyond $1000 \mathrm{HV}_{1}$ which five times as much as untreated samples near the surface. Then it quickly reduces when then distance ranges from $70 \mathrm{um}$ to $90 \mathrm{um}$. At last, it becomes the same with the hardness of untreated samples. Nitrided samples at $460^{\circ} \mathrm{C}$ and $500^{\circ} \mathrm{C}$ have nearly the same tendency of hardness.

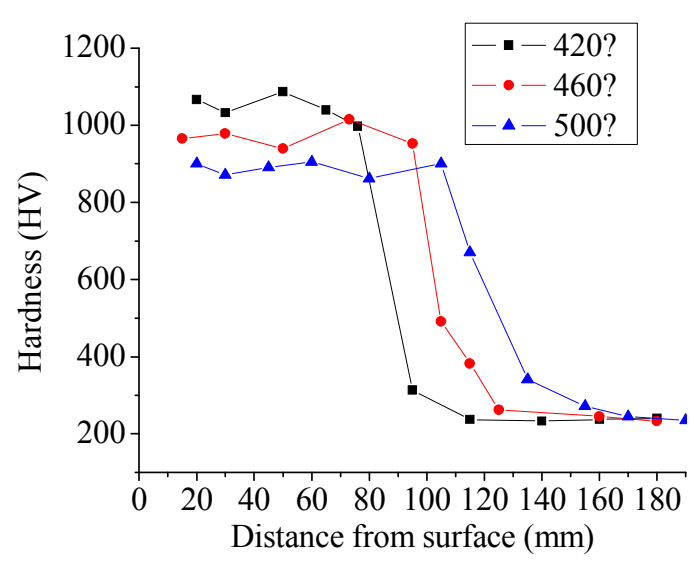

(a)

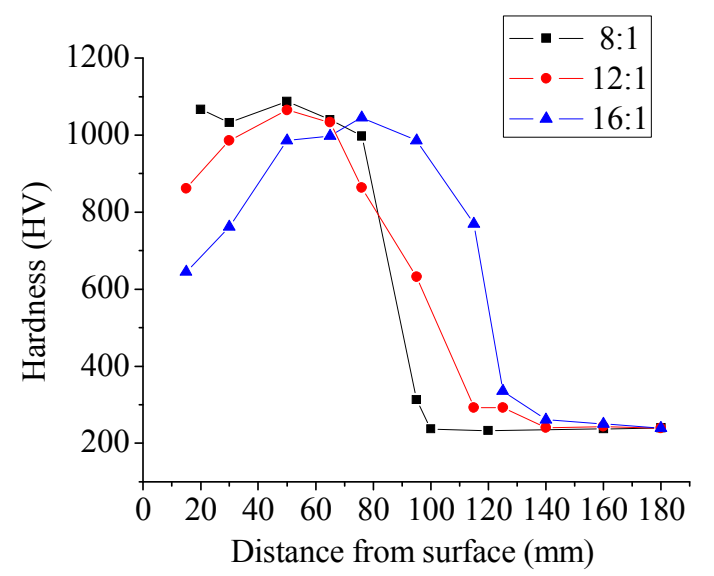

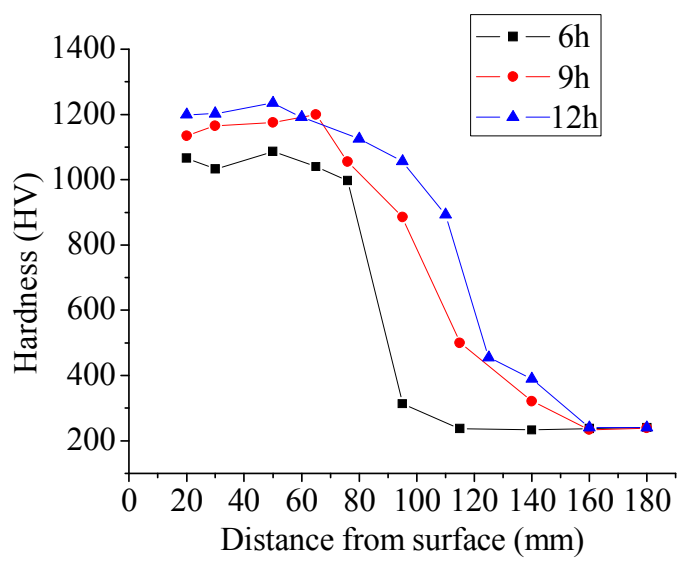

(b)

(c)

Fig 1 Hardness of plasma nitrided matensitic stainless steel in different conditions 
The Fig 1(b) shows the hardness of the nitrided layer at different time. Hardness of the nitrided layer is uniform and was enhanced with the time increases. But the hardness changes less when time ranges from $9 \mathrm{~h}$ to $12 \mathrm{~h}$.

Hardness of the nitrided layer at different gas proportions is showed in Fig 1(c). The tendency of hardness changes when the gas proportion is greater than 8:1. Hardness of nitrided layer decreases where is $20 \mathrm{um}$ from the surface and it is about $620 \mathrm{HV}_{1}$ and $830 \mathrm{HV}_{1}$ while the gas proportions are 12:1 and 16:1. Hardness rises with distance ranges from 20um to 50um, then reaches the best and begins to reduce as the changes of hardness at proportion of $8: 1$.

Microstructure of nitrided layer. Fig 2 showes the metallographic of the nitriding layers of different conditions on 410 martensitic stainless steel and a, b, c, d, e, f and g respectively are samples of $1,2,3,4,5,6$, and 7 . It is obvious that the matensitic stainless steel has a nitrided layers

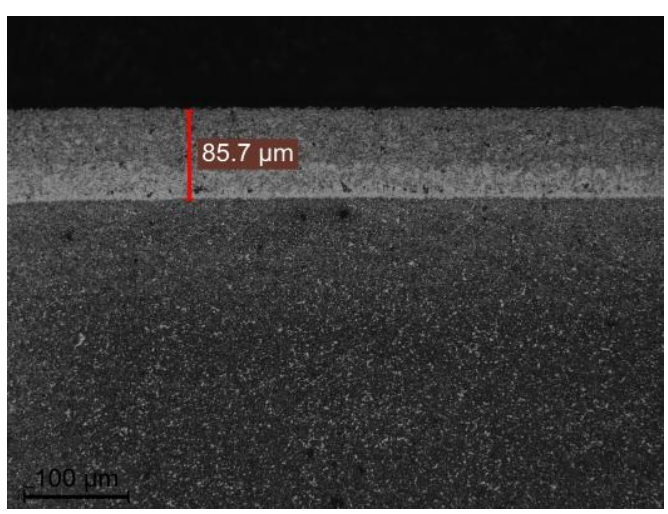

(a)

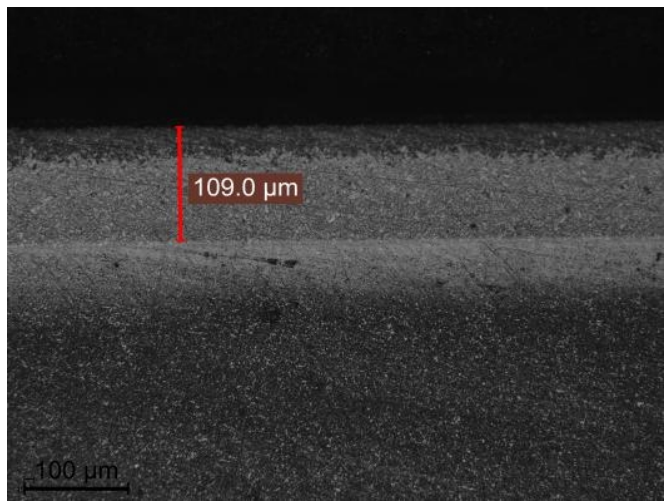

(c)

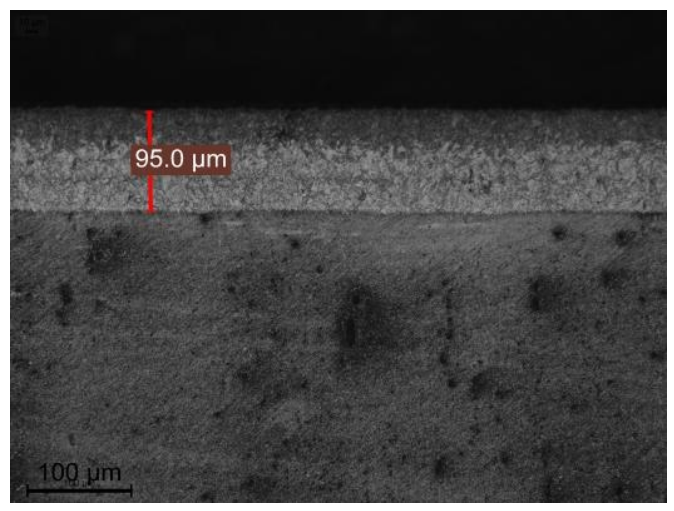

(e)

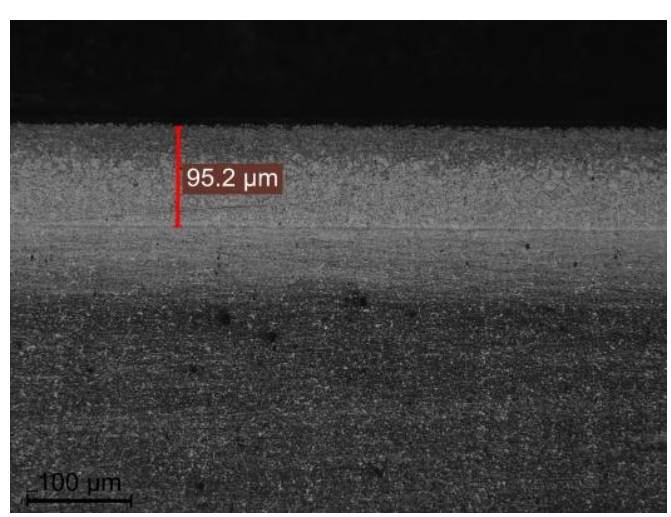

(b)

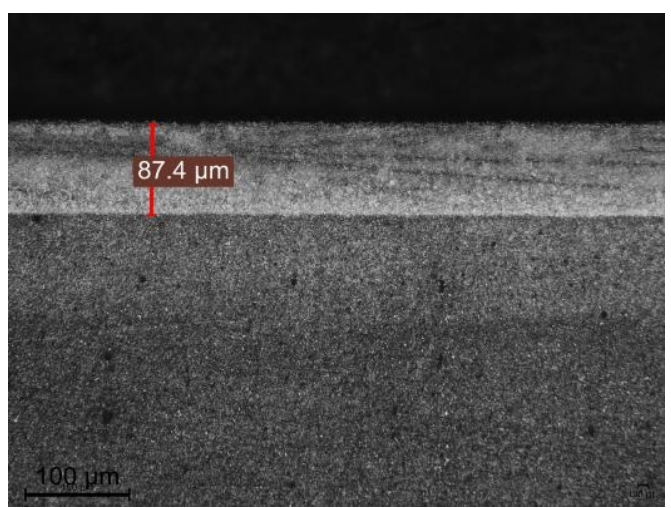

(d)



(f) 


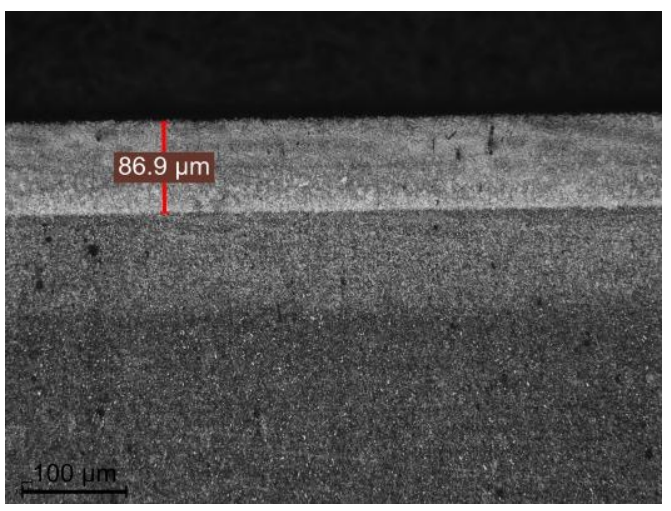

(g)

Fig 2 Microstructure of nitrided matensitic stainless steel in different conditions and the thickness of layer improves with the temperature and time increase. But the thickness increases at first and then almost keeps the same when gas proportions grow.

\section{Conclusions}

(1)The hardness of AISI410 stainless steel decreases and thickness of nitrided layer improves. The hardness becomes $1100 \mathrm{HV}_{1}$ at $420^{\circ} \mathrm{C}$.

(2)The hardness and thickness of nitrided layer both improve with the time increases.

(3)Hardness of nitrided layer decreases where is $20 \mathrm{um}$ from the surface and it is about $620 \mathrm{HV}_{1}$ and $830 \mathrm{HV}_{1}$ while the gas proportions are 12:1 and 16:1. At the same time, the thickness increases at first and then almost keeps the same when the gas proportions grower.

\section{References}

[1] Menthe E, Bulak A, Olfe J, et al. Surface and Coating Technology, 2000, 1133- 1134: 259- 263.

[2] Sun Y, Bell T. Wear, 1998, 218( 1) : 34- 42.

[3] WANG Liang, XU Bin, YU Zhiwei, et al. Surf ace and Coating Technology, 2000, 130: 304308.

[4] GOU Wei , LI Guoqing, LIU Cui, et al. Trans Mater Heat Treat , 2004, 25( 4) :57- 59.

[5] WANG Liang, XU Xiaolei, XU Bin, et al. Tribology, 2000, 20(1) : 67- 69. 